\title{
IRS2 variants and syndromes of severe insulin resistance
}

\author{
W. E. Bottomley • M. A. Soos • C. Adams • T. Guran • T. A. Howlett • A. Mackie • \\ J. Miell • J. P. Monson • R. Temple • Y. Tenenbaum-Rakover • J. Tymms • \\ D. B. Savage • R. K. Semple • S. O'Rahilly • I. Barroso
}

Received: 19 November 2008 / Accepted: 16 February 2009 / Published online: 18 April 2009

(C) The Author(s) 2009. This article is published with open access at Springerlink.com

Keywords Diabetes · Genetics · Insulin resistance $\cdot$ IRS2

To the Editor: Numerous lines of experimental evidence implicate IRS2 in insulin signal transduction in key insulinresponsive tissues, and the combination of insulin resistance and pancreatic beta cell failure of homozygous $\operatorname{Irs} 2$ knockout mice is highly reminiscent of both core abnormalities in human type 2 diabetes [1]. IRS2 is therefore a compelling

Electronic supplementary material The online version of this article (doi:10.1007/s00125-009-1345-4) contains supplementary material,

which is available to authorised users.

W. E. Bottomley $\cdot$ I. Barroso $(\square)$

Wellcome Trust Sanger Institute,

Hinxton,

Cambridge CB10 1SA, UK

e-mail: ib1@sanger.ac.uk

M. A. Soos - C. Adams • D. B. Savage - R. K. Semple •

S. O'Rahilly

University of Cambridge Metabolic Research Laboratories,

Institute of Metabolic Science, Addenbrooke's Treatment Centre,

Addenbrooke's Hospital,

Cambridge, UK

T. Guran

Department of Pediatric Endocrinology and Diabetes,

Marmara University,

Istanbul, Turkey

T. A. Howlett

Department of Diabetes and Endocrinology,

Leicester Royal Infirmary,

Leicester, UK candidate gene for involvement in prevalent forms of type 2 diabetes and insulin resistance in humans. Nevertheless, genetic studies to date in humans suggest that common polymorphisms found in the IRS2 coding or promoter regions are not associated with insulin resistance or type 2 diabetes [2-4]. Furthermore, these studies have revealed few
J. Miell

Department of Endocrinology, University Hospital Lewisham, London, UK

J. P. Monson

Centre for Endocrinology, William Harvey Research Institute, St Bartholomew's and The Royal London Hospitals, QMUL, London, UK

R. Temple

Elsie Bertram Diabetes Centre,

Norfolk and Norwich University Hospital NHS Trust,

Norwich, UK

Y. Tenenbaum-Rakover

Pediatric Endocrine Unit, Ha'Emek Medical Center, Afula, Israel

\section{J. Tymms}

Diabetes Centre, Royal Albert Edward Infirmary,

Wigan, UK 
rare non-synonymous variants, none of which appear to have a strong impact on insulin sensitivity $[5,6]$.

We have assembled a large cohort of patients with severe insulin resistance that is likely to be highly enriched for monogenic disorders of insulin action. All participants gave informed consent, and our investigations were carried out with the approval of the local research ethics committee in Cambridge, UK. This strategy has previously been validated, for example, by the finding of a convincing pathogenic mutation in $A K T 2$ [7], despite a failure to find association between common genetic variation in $A K T 2$ and metabolic traits [8]. We have now applied this robust approach to IRS2.

We sequenced the coding region of IRS2 in 161 predominantly Europid $(n=114)$ patients with severe insulin resistance. Severe insulin resistance was defined as (1) a fasting insulin level of $>150 \mathrm{pmol} / 1$ or a peak insulin level on oral glucose tolerance testing of $>1,500 \mathrm{pmol} / 1$ in those without diabetes and a BMI of $<30 \mathrm{~kg} / \mathrm{m}^{2}$, or (2) an exogenous insulin requirement of $>3 \mathrm{U} / \mathrm{kg} /$ day in those with complete insulin deficiency and a BMI of $<30 \mathrm{~kg} / \mathrm{m}^{2}$. Those with partial beta cell decompensation or a BMI of $>30 \mathrm{~kg} / \mathrm{m}^{2}$ were included at the investigator's discretion based on clinical and biochemical features of insulin resistance disproportionate to body weight, determined by reference to sex and BMI-specific 95th percentiles for plasma insulin from more than 500 non-diabetic volunteers.

Eight rare, novel, non-synonymous variants in the IRS2 gene were identified in eight patients from this cohort (designated as patients SIR1 to SIR8 in Table 1), four of whom are Europids. Wherever possible, family members of probands with non-synonymous variants were studied to look for co-segregation of the genetic variant with insulin resistance or diabetes.

In the case of SIR1 (c.233G $>$ A, p.S78N), four of the five family members studied were found to be heterozygous for this mutation and had insulin levels commensurate with their degree of obesity [9] (fasting insulin levels 45$95 \mathrm{pmol} / \mathrm{l}$, BMI $30-38.8 \mathrm{~kg} / \mathrm{m}^{2}$ ); hence, there was no clear co-segregation of the mutation with severe insulin resistance in the family (Electronic supplementary material [ESM] Fig. 1), indicating that the $\mathrm{S} 78 \mathrm{~N}$ change in IRS2 within this family is either benign, as suggested by Polyphen (http://genetics.bwh.harvard.edu/pph, accessed 4 March 2008)/SIFT (http://blocks.fhcrc.org/sift/SIFT.html, accessed 22 December 2008) analysis, or is not the sole genetic determinant of insulin resistance within the kindred. The location of serine 78 within the Pleckstrin homology domain of the protein (ESM Fig. 2) and its strong evolutionary conservation support the view that a pathogenic role for this protein-altering variant should not be entirely discounted on the basis of these genetic data.

Eight members of the Turkish family of SIR3, with five affected members, were also studied, but only the unaffect- ed father of the proband was found to carry the same heterozygous mutation (c.1570A $>$ G, p.I524V). Thus, this non-synonymous change in IRS2 convincingly fails to cosegregate with insulin resistance in this family (ESM Fig. 3, ESM Table 1) and is thus most probably benign.

Of the four available relatives of Ashkenazy proband SIR4 (c.2485C > T, p.P829S), three were heterozygous for this variant, two of whom exhibited elevated fasting insulin levels with respect to 500 non-diabetic volunteers (ESM Fig. 4). However, incomplete clinical data precluded a definitive conclusion regarding co-segregation of the mutation with the phenotype in this family. It is of interest that this variant was absent in 185 Ashkenazy controls and that proline 829 is conserved in a diverse range of species, from Pan troglodytes (chimpanzee) down to Xenopus tropicalis (Western clawed frog) (ESM Fig. 5).

The non-synonymous variant found in SIR6 (c.2834C $>\mathrm{T}$, p.S945F) was found to be absent in this patient's mother, who presented with a similar but milder syndrome of partial lipodystrophy. Although the father of SIR6 was unavailable for study (ESM Fig. 6), this suggests that this variant does not underlie the observed phenotype.

In a study limited to the parents of SIR8 (ESM Fig. 7), there appeared to be co-segregation of the non-synonymous variant (c.3983A $>$ G, p.H1328R) found in the proband with clinical phenotype, but because this change was also observed twice on sequencing additional controls, this variant was deemed to be benign.

Subsequent to our family studies, we also sequenced the IRS2 gene in 173 Europid controls (mean fasting insulin $30 \mathrm{pmol} / 1$, mean BMI $27.3 \mathrm{~kg} / \mathrm{m}^{2}$ ) to assess possible enrichment of non-synonymous variants in the patients with severe insulin resistance relative to insulin-sensitive controls. In the controls (Table 1), we detected four non-synonymous heterozygous variants, one of which (c.3983A $>$ G, p.H1328R) was present in two controls (control 1 and 4), as well as in proband SIR8. These findings suggest that there is no significant difference in the frequency of non-synonymous variants in the IRS2 gene between the two groups studied, with four non-synonymous variants being found in each group of Europids (4/114 patients vs $4 / 173$ controls, $p=0.54$ ).

Our study has revealed several new non-synonymous variants in the IRS 2 gene in both a cohort of predominately Europid individuals with severe insulin resistance and in insulin-sensitive Europid volunteers. Wherever possible, we have performed co-segregation studies on the available family members of severely insulin-resistant patients, but we have found no clear evidence that any of the nonsynonymous variants studied has a fully penetrant pathogenic effect. Given the difficulty involved in performing detailed functional evaluation of each variant, we thought to describe these results in the hope that if others have similar 


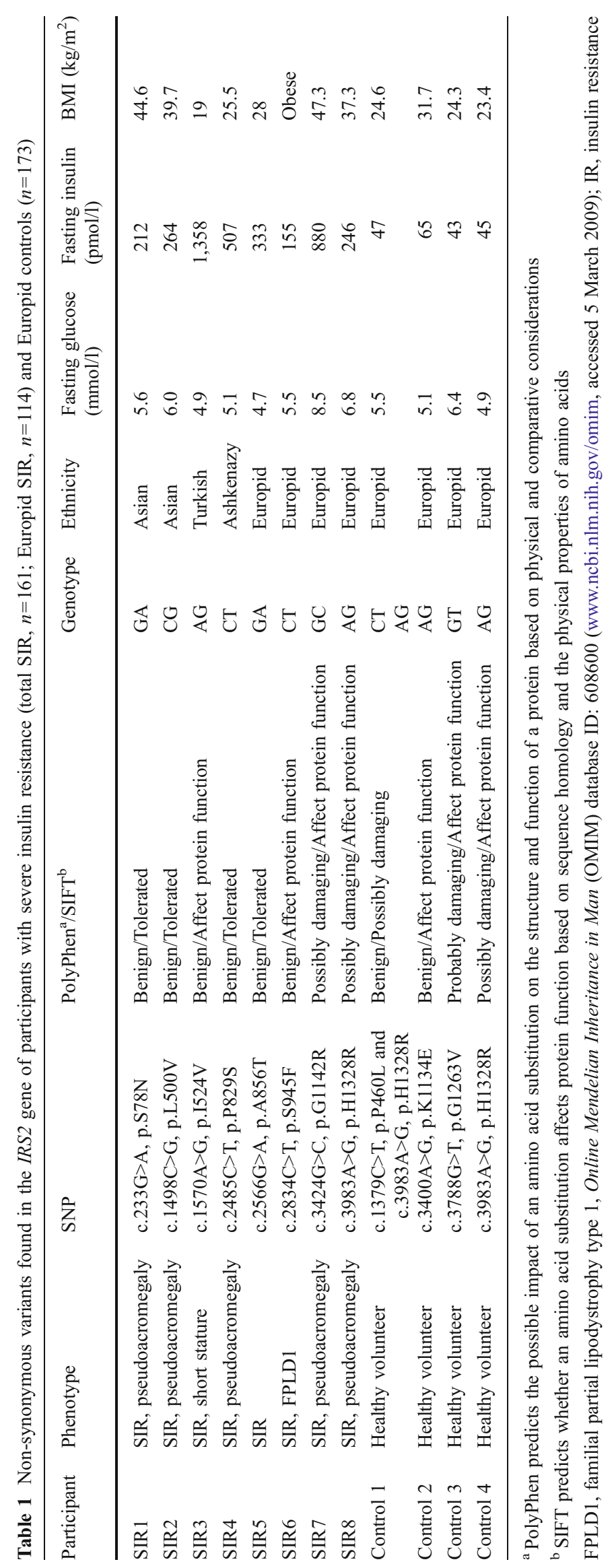


data, aggregate data may provide the impetus required to carry out these additional analyses. However, we cannot rule out the possibility that some of these variants contribute to the phenotype of these patients in combination with environmental or other, unknown, genetic variants. Sequencing of IRS2 in insulin-sensitive controls demonstrated that there was no enrichment in the number of nonsynonymous variants among the cases, but because this study only had $4.85 \%$ power (at $p=0.05$ significance) to detect the modest differences in frequency observed here, much larger studies will be required to elucidate the role of rare IRS 2 variants in disease. To obtain nominal levels of significance $(p=0.05)$, assuming the same proportions of mutation carriers and non-carriers as those we report here, the study would need to include at least tenfold more insulin-sensitive and insulin-resistant participants.

In conclusion, we have identified several novel mutations in the IRS2 gene, which, despite no clear segregation with insulin resistance in this study, merit further investigation in additional cohorts; some variants (e.g. P829S) may yet be shown to have an effect on insulin sensitivity in humans.

Acknowledgements This study was supported by grants from the Wellcome Trust (R. K. Semple, Intermediate Clinical Fellowship 080952/Z/06/Z; S. O’Rahilly, Programme Grant 078986/Z/06/Z; I. Barroso, Wellcome Trust grant 077016/Z/05/Z), GlaxoSmithKline (D. B. Savage, GlaxoSmithKline Clinical Fellowship) and the UK National Institute for Health Research (NIHR) Cambridge Biomedical Research Centre. The authors would also like to thank A. Thompson and A. Daly from the Wellcome Trust Sanger Institute for technical and informatics support, respectively.

Duality of interest The authors declare that there is no duality of interest associated with this manuscript.
Open Access This article is distributed under the terms of the Creative Commons Attribution Noncommercial License which permits any noncommercial use, distribution, and reproduction in any medium, provided the original author(s) and source are credited.

\section{References}

1. Withers DJ, Gutierrez JS, Towery H et al (1998) Disruption of IRS-2 causes type-2 diabetes in mice. Nature 391:900-904

2. Bernal D, Almind K, Yenush L et al (1998) Insulin receptor substrate-2 amino acid polymorphisms are not associated with random type2 diabetes among Caucasians. Diabetes 47:976-979

3. Wang H, Rissanen J, Miettien R et al (2002) New amino acid substitutions in the IRS-2 gene in Finnish and Chinese subjects with late-onset type2 diabetes. Diabetes 50:1949-1951

4. Iwamoto K, Mori H, Okazawa H, Hashiramoto M, Kasuga M (2002) Identification of a single nucleotide polymorphism showing no insulin-mediated suppression of the promoter activity in the human insulin receptor substrate 2 gene. Diabetologia 45:1182-1195

5. Almind K, Frederiksen SK, Bernal D et al (1999) Search for variants of the gene-promoter and the potential phosphotyrosine encoding sequence of the insulin receptor substrate-2 gene: evaluation of their relation with alterations in insulin secretion and insulin sensitivity. Diabetologia 42:1244-1249

6. D'Alfonso R, Marini MA, Frittitta L et al (2003) Polymorphisms of the insulin receptor substrate- 2 in patients with type 2 diabetes. J Clin Endocrinol Metab 88:317-322

7. George S, Rochford JJ, Wolfrum C et al (2004) A family with severe insulin resistance and diabetes due to a mutation in $A K T 2$. Science 304:1325-1328

8. Tan K, Kimber WA, Luan J et al (2007) Analysis of genetic variation in Akt2/PKB- $\beta$ in severe insulin resistance, lipodystrophy, type 2 diabetes, and related metabolic phenotypes. Diabetes 56:714-719

9. Savage DB, Agostini M, Barroso I et al (2002) Digenic inheritance of severe insulin resistance in a human pedigree. Nat Gen 31 : 379-384 